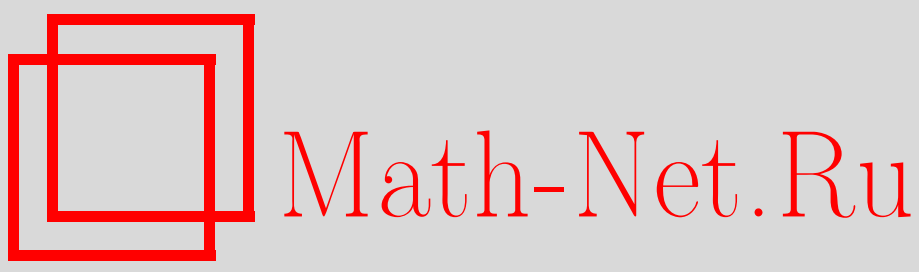

П. И. Топалов, Вычисление тонкого инварианта Фоменко-Цишанга для основных интегрируемых случаев движения твердого тела, Матем. сб., 1996, том 187, номер 3, 143-160

DOI: https://doi.org/10.4213/sm120

Использование Общероссийского математического портала Math-Net.Ru подразумевает, что вы прочитали и согласны с пользовательским соглашением

http://www . mathnet.ru/rus/agreement

Параметры загрузки:

IP : 34.229 .45 .116

26 апреля 2023 г., 17:58:07 
УДК 515.1

\author{
П. Топалов
}

\title{
Вычисление тонкого инварианта Фоменко-Цишанга для основных интегрируемых случаев движения твердого тела
}

\author{
В работе дается общий методвычисления меток топологического инварианта. \\ Библиография: 5 названий.
}

\section{1. Введение}

Пусть $\left(M^{4}, \omega\right)$ - симплектическое многообразие с симплектической структурой $\omega$. Пусть на нем задана гамильтонова система $\vec{v}=\operatorname{sgrad} H$ с гамильтонианом $H \in C^{\infty}(M)$, нерезонансная и интегрируемая по Лиувиллю с помощью боттовского интеграла $f \in C^{\infty}(M)$ (см. [1], [2]). Для тонкой топологической классификации таких систем в работе [1] А. Т. Фоменко и Х. Цишанг открыли тонкий топологический инвариант $W^{*}\left(Q^{3}, v\right)$, названньй меченой молекулой, который классифицирует послойно эквивалентные системы на фиксированных и регулярных изоэнергетических поверхностях $Q_{h}^{3} \stackrel{\text { def }}{=}\{H(x)=h\}$. Меченая молекула является графом, вершины и ребра которого снабжены некоторыми метками [2].

В работе [2] А. В. Болсинов, С. В. Матвеев и А.Т. Фоменко доказали теорему реализуемости, т.е. что любая абстрактно заданная "меченая молекула", снабженная метками, удовлетворяющими естественным условиям, полностью описанньм в [2], является инвариантом $W^{*}\left(Q_{h}^{3}, \vec{v}\right)$ для некоторой интегрируемой, невырожденной гамильтоновой системы $\vec{v}$ на подходящем 3 -многообразии $Q_{h}^{3}$. Мы будем далее пользоваться терминологией и обозначениями работы [2].

Обычно в физике и механике возникает следуюшая задача. Пусть даны две интегрируемые гамильтоновые системы. Надо узнать эквивалентны ли они в тонком топологическом смысле, т.е. существует ли гомеоморфизм, отображающий слоение Лиувилля одной системы в слоение Лиувилля другой. В большинстве случаев практически единственный способ решить эту задачу состоит в вычислении соответствуюших инвариантов Фоменко-Цишанга.

Метки инварианта $W^{*}\left(Q_{h}^{3}, v\right)$ бывают двух типов. Первый тип это $\varepsilon$ - и $r$-метки. Они являются “локальньми" инвариантами и стоят на ребрах молекулы. Второй тип метки, $n$-метки, имеют "глобальный” характер. Эти числа являются аналогом числа Эйлера для комплексных линейных расслоений над двумерными поверхностями. Для построения молекул и подсчета $r$-меток уже сушествует развитая техника (см. [3], [4]).

В настоящей работе найдены явные соотношения между группой гомологий $H_{1}\left(Q^{3}\right)$ и метками инварианта $W^{*}\left(Q^{3}, v\right)$. Так как в механике и физике обычно 
топология 3-многообразия $Q^{3}$ известна изначально (или ее легко можно найти), то таким образом получается весьма эффективный способ нахождения $n$-меток. В конце работы в качестве применения развитой нами техники мы полностью вычисляем инвариант Фоменко-Цишанга для некоторых важных интегрируемых случаев движения твердого тела и, следовательно, получаем тонкую топологическую классификацию всех этих динамических систем.

\section{2. Основные определения и результаты}

Пусть $G$ - молекула (см. [1], [2]). “Семњей” молекулы $G$ называется связньй подграф графа $G$, вершины которого отличны от атома $A$ и на всех его ребрах $r=\infty$. Все остальные ребра в $G$, которые имеют вершину, принадлежашую семье, должны иметь $r$-метку, отличную от $\infty$.

Иначе можно сказать так: "сотрем" в $G$ все ребра, на которых $r \neq \infty$. Тогда, очевидно, $G$ распадается на связные куски. Все такие куски, которые не содержат атомов $A$, будут “семьями”.

Из оставшихся кусков рассмотрим те, которые отличны от атомов $A$. В них существуют ребра, одна из вершин которых есть атом $A$. Для любого такого куска фиксируем раз и навсегда одно и только одно такое ребро. Множество всех таких ребер будем называть “множеством фиксированных ребер”.

ОпредЕлЕниЕ 1. Ребро молекулы $G$ называется “существенньп”, если оно не принадлежит множеству фиксированных ребер и если обе его вершины не принадлежат семьям.

ОПРЕДЕЛЕНИЕ 2. “Энергией семьи” $F \subset G$ будем называть число

$$
\begin{gathered}
E(F) \stackrel{\text { def }}{=} \sum_{I_{0}}\left(-\frac{\gamma(e)}{\alpha(e)}\right)+\sum_{I_{1}} \frac{\alpha(e)}{\beta(e)}+\sum_{I_{2}}\left(-\frac{\delta(e)}{\beta(e)}\right)+\sum_{I_{3}}\left(\frac{\alpha(e)}{\beta(e)}-\frac{\delta(e)}{\beta(e)}\right) \\
=n(F)+\sum_{I_{1} \sqcup I_{3}} \varepsilon(e) r(e)+\sum_{\left(I_{2} \sqcup I_{3}\right) \backslash\{e \mid r(e)=0\}} \varepsilon(e)(r(-e)-1),
\end{gathered}
$$

где

$e \in G$ - ориентированное ребро молекулы,

$\left[\begin{array}{ll}\alpha(e) & \beta(e) \\ \gamma(e) & \delta(e)\end{array}\right]$ - матрица склейки (см. [2]),

$n(F)-n$-метка,

$r(e)-r$-метка,

$I_{0}=\{e \in F\}$,

$I_{1}=\left\{e \in G \mid v_{0}(e) \in F, v_{1}(e) \notin F\right\}$ - "выходящие" ребра,

$I_{2}=\left\{e \in G \mid v_{0}(e) \notin F, v_{1}(e) \in F\right\}$ - "входящие" ребра,

$I_{3}=\left\{e \notin F \mid v_{0}(e) \in F, v_{1}(e) \in F\right\}$ - "петли",

$v_{0}(e)$ - начало ребра $e$,

$v_{1}(e)$ - конец ребра $e$,

$(-e)$ - ребро е с обратной ориентацией.

По поводу определений молекул, атомов, $r$-меток, $n$-меток и $\varepsilon$-меток см. работу [2]. 
ЗАмечание 1. Энергия семьи $E(F)$, очевидно, является тонким топологическим инвариантом гамильтоновой системы (так как $E(F)$ выражается через метки). С другой стороны, легко проверяется, что $E(F)$ не зависит от ориентации ребер молекулы. При изменении ориентации $Q^{3}$ энергия семьи $E(F)$ меняет знак.

Для любого графа $G$ без звездочек (см. [2]) определим "полную энергию кручения", которая является цельм числом, определенньм с точностью до знака, которое мы будем обозначать через $N(G)$.

I) Для графа

$$
G \stackrel{\text { def }}{=}\left(A_{1} \longleftarrow W_{0} \longrightarrow A_{2}\right),
$$

если $\beta_{01}, \beta_{02} \neq 0$, то по определению считаем, что

$$
N(G) \stackrel{\text { def }}{=} \beta_{01} \beta_{02} E(F),
$$

где $E(F)$ - энергия единственной семьи молекулы.

II) Для графа

$$
G \stackrel{\text { def }}{=}\left(\begin{array}{c}
A_{4} \longleftarrow \begin{array}{c}
W_{1} \\
n_{1}
\end{array} \\
\end{array} \begin{array}{c}
W_{2} \\
n_{1}
\end{array} \longrightarrow A_{3}\right),
$$

если $\beta_{12}, \beta_{23}, \beta_{14} \neq 0$, то имеет место формула

$$
N(G) \stackrel{\text { def }}{=} \beta_{14} \beta_{23} \beta_{12}\left[E\left(F_{1}\right) E\left(F_{2}\right)-\frac{1}{\beta_{12}^{2}}\right]
$$

где $E\left(F_{i}\right)$ - энергия семьи $F_{i}$.

III) Для графа

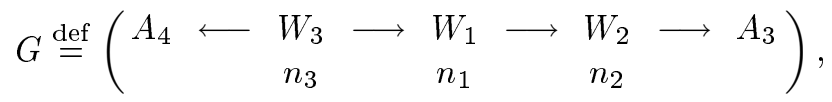

если $\beta_{34}, \beta_{31}, \beta_{12}, \beta_{23} \neq 0$, то тогда

$$
N(G) \stackrel{\text { def }}{=} \beta_{34} \beta_{31} \beta_{12} \beta_{23}\left[E\left(F_{1}\right) E\left(F_{2}\right) E\left(F_{3}\right)-\frac{E\left(F_{2}\right)}{\beta_{31}^{2}}-\frac{E\left(F_{3}\right)}{\beta_{12}^{2}}\right] .
$$

IV) Для графа

$$
G \stackrel{\text { def }}{=}\left(\begin{array}{c}
A_{2} \\
\uparrow \\
\subset W_{1} \\
\downarrow \\
A_{3}
\end{array}\right),
$$

если $\beta_{11}, \beta_{12}, \beta_{13} \neq 0$, то

$$
N(G) \stackrel{\text { def }}{=} \beta_{11} \beta_{12} \beta_{13}\left[E\left(F_{1}\right)-\frac{2}{\beta_{11}}\right] .
$$


Здесь через $\beta_{i j}$ обозначены знаменатели $r$-меток ребер $e_{i j}$ (начальная вершина (буква) ребра $e_{i j}$ имеет индекс $i$, а вторая вершина имеет индекс $j$ ).

Для произвольного графа $G$, не имеюшего звездочек, определение $N(G)$ будет дано в процессе доказательства основной теоремы.

В случае, когда молекула $G$ содержит звездочки, поступим следующим образом. Любой букве $W^{*}$ со звездочкой сопоставим новую букву $\widetilde{W}$ (см. рис. 1 ) так, чтобы выполнялись следующие условия.

1) $q(\widetilde{W})=q\left(W^{*}\right)+\left\{\right.$ число звездочек в $\left.W^{*}\right\}$, где $q(W)-$ валентность буквы $W$.

2) $g(\widetilde{W})=g\left(W^{*}\right)$, где $g(W)$ - род буквы $W$.

3) Все вторые вершины новых ребер будут буквами $A$ (эти ребра ориентируем по направлению к ним).

4) На всех новых ребрах $r=1 / 2, \varepsilon=1$.

5) Годится любая буква $\widetilde{W}$, для которой выполняются условия 1$)-4)$.

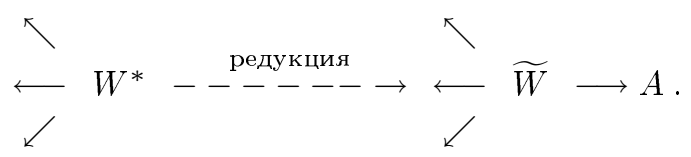

Рис. 1

Проделывая эти операции для любой буквы со звездочками графа $G$, получим новый граф $\widetilde{G}$. Все $\varepsilon-, r$ - и $n$-метки молекулы $\widetilde{G}$ останутся такими же, как и у $G$.

ОпРЕДЕЛЕНИЕ 3 . Если молекула $G$ имеет звездочки, то положим

$$
N(G) \stackrel{\text { def }}{=} N(\widetilde{G})
$$

ЗАмечАниЕ 2. Несмотря на неоднозначность выбора букв $\widetilde{W}$, это определение корректно.

Теорема 1 (основная). 1) Пусть $G$ - молекула. Тогда

$$
\begin{aligned}
& \operatorname{rk}\left(H_{1}\left(Q^{3}\right)\right) \geqslant b_{1}(G)+2 \sum_{W \neq A} g(W), \\
& \operatorname{rk}\left(H_{1}\left(Q^{3}\right)\right) \leqslant\left(b_{1}(G)+2 \sum_{W \neq A} g(W)\right)+\sum_{W \neq A} q(W),
\end{aligned}
$$

где $q(W)$ - валентность атома $W, b_{1}(G)$ - первое число Бетти графа $G, g$ - род базы буквы $W$.

2) Любое существенное ребро е дает в $H_{1}\left(Q^{3}\right)$ прямое слагаемое $Z_{\beta(\text { e) }}$. Если атом не принадлежит никакой семье, то все его звездочки дают независимые прямые слагаемые $Z_{2}$. 
3) Для любой молекульи имеют место соотношения:

$$
N(G)= \pm \begin{cases}0, & \text { если } \operatorname{rk} H_{1}\left(Q^{3}\right)>b_{1}(G)+2 \sum_{W \neq A} g(W) \\ \operatorname{card}\left(\operatorname{Tor} H_{1}\left(Q^{3}\right)\right), & \text { иначе. }\end{cases}
$$

Эти соотношения будем называть “"

Приведем теперь некоторые следствия.

СлЕДСТВИЕ 1. Если $\operatorname{rk} H_{1}\left(Q^{3}\right)<2$, то все атомы плоские $($ m.e. $g(W)=0)$. В частности, все атомы на гомологических 3-сферах и гомологических проективных 3-пространствах являются плоскими.

СлеДСТВИЕ 2. Если $Q^{3} \cong\left\{S^{3}, S^{1} \times S^{2}, T^{3}\right.$ u их связные суммы $\}$, то на существенных ребрах стоят метки 0 и $о$. Для $Q^{3} \cong \mathbb{R P}^{3}$ на существенных ребрах стоят метки 0 или 1/2. При этом метка $1 / 2$ стоит только на одном мecme.

СЛЕДСТВИЕ 3. Если граф не имеет семьи, то знаменатели конечных $r$-меток не превосходят $\operatorname{card}\left(\operatorname{Tor}\left(H_{1}\left(Q^{3}\right)\right)\right)$.

\section{3. Доказательство основной теоремы}

Вершины графа, которые отличны от атома $A$, будем обозначать через $W$ (быть может, с индексом). Пока будем предполагать, что зейфертова реализация буквы $W$ не имеет особых слоев Зейферта (случай наличия особых слоев легко редуцируется к этому). Следовательно,

$$
W=\left(F_{g}, q\right)
$$

где поверхность $F_{g}$ - двумерная, ориентируемая, замкнутая, связная и компактная рода $g$, а $q$-валентность атома $W$ или, что тоже самое, число дырок в $F_{g}$. В этом случае, очевидно, имеем:

$$
W=F_{g} \times S^{1}
$$

и сечение $F_{g} \times\{1\}$ является "допустимым" (определение допустимого сечения см. в [2]). Пусть граф̆ $\mathrm{G}$ имеет вершины $\left\{W_{1}, \ldots, W_{k}, A_{1}, \ldots, A_{s}\right\}$, где $A_{i}$ есть атом $A$. Ребра графа разбиваем на "внутренние" и "внешние".

ОПРЕДЕЛЕНИЕ 4. Ребро назьвается “внутренним”, если его концы отличны от атома $A$ (например, петля всегда является внутренним ребром). Если один из концов ребра есть атом $A$, то ребро является "внешним".

На каждой вершине $W$ мы выбираем допустимые координаты (см. [2]) в группе гомологий $H_{1}(\partial W)$. Край $\partial W$, очевидно, состоит из $q$ несвязных экземпляров тора $T^{2}$. На краях полноторий $A$ выбираем допустимые координаты так: цикл $\lambda$ гомологичен нулю (при вложении $\partial A \subset A$ ), а цикл $\mu$-ориентируемая потоком $\operatorname{sgrad} H$ критическая окружность (мы, конечно, считаем, что $A \subset Q^{3}$ ). Цикл $\lambda$ на 

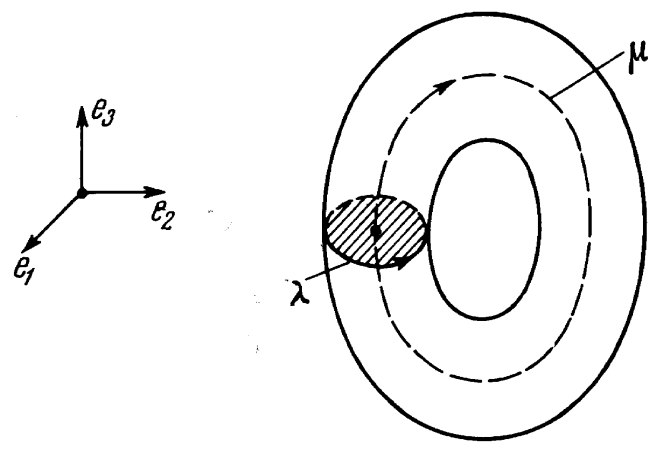

Рис. 2

$\partial A$ ориентируем так, чтобы его ориентация индуцировалась ориентацией базы, которая, в свою очередь индуцируется ориентацией $Q^{3}$ и потока sgrad $H$ (см. рис. 2).

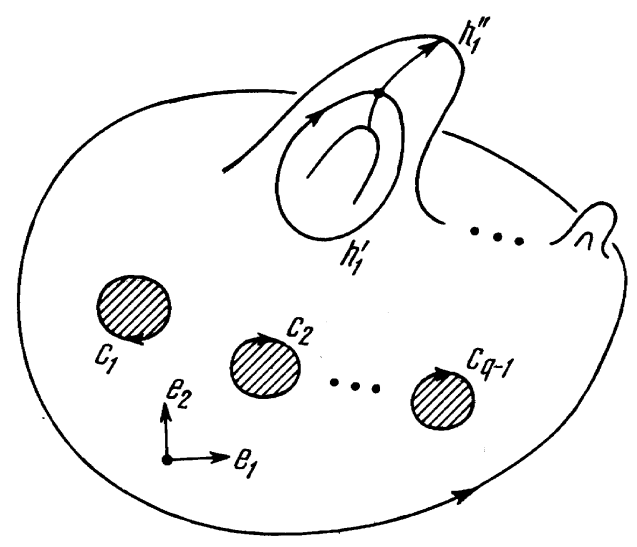

Рис. 3

Так как в нашей ситуации $W=F_{g} \times S^{1}$, то из формулы Кюннета имеем:

$$
H_{1}(W) \cong H_{1}\left(F_{g}\right) \oplus Z
$$

Координаты в $H_{1}(W)$ выбираем следуюшим образом.

а) В качестве $\lambda$ выбираем слой расслоения Зейферта, ориентированный потоком $\operatorname{sgrad} H$.

б) Ориентируем (естественным образом) базу $F_{g}$ и выберем в группе гомологий $H_{1}\left(F_{g}\right)$ базисные циклы так, как показано на рис. 3 . Упорядочим базис в $H_{1}(W)$ 
следующим образом:

$$
\left(c_{1}, \ldots, c_{q-1} ; \lambda ; h_{1}^{\prime}, h_{1}^{\prime \prime}, \ldots, h_{g}^{\prime}, h_{g}^{\prime \prime}\right) .
$$

На краях $\partial W=\bigsqcup_{i=1}^{q} T_{i}^{2}$ выберем координаты $\left(\bar{\lambda}_{i}, \bar{c}_{i}\right)$ так, чтобы при вложении $T_{i}^{2} \subset W$ цикл $\bar{\lambda}_{i}$ был гомологичен $\lambda$, и $\bar{c}_{i}$ был гомологичен $c_{i}$.

На полноториях $A_{i}$ берем координату $e_{i}-$ ориентированную потоком $\operatorname{sgrad} H$ критическую окружность.

Любое внутреннее ребро графа $G$ мы заменим на два внутренних ребра и новую вершину $K=T^{2} \times I$ так, как показано на рис. 4 (это техническая операция позволяет применить единый подход ко всем графам),

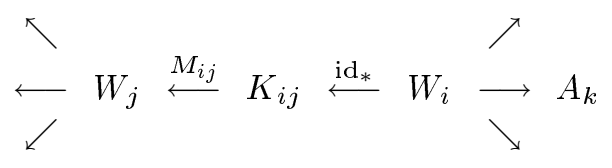

Рис. 4

где $M_{i j}$ - матрица склейки.

Координаты в $H_{1}(K)$ индуцируем вложением $\partial W \ni T^{2} \subset K$ и обозначим $\left(k^{\prime}, k^{\prime \prime}\right)$. Таким образом, координаты на одном куске $\partial K$ уже выбраны, а на другом куске (торе) индуцируем координаты вложением $\partial K \ni T^{2} \subset K$. Если ребро $e$ связывает вершины $W_{1}$ и $W_{2}$ именно в такой последовательности в графе $G$, то возникаюшие при описанной выше процедуре два тора мы будем обозначать через $T_{12}^{(1)}$ и $T_{12}^{(2)}$ соответственно, а их базис через $\left(\lambda_{12}^{(i)}, \mu_{12}^{(i)}\right)$, где $i=1,2$. Кольцо $K$ будем обозначать через $K_{12}$, а его базис через $\left(k_{12}^{\prime}, k_{12}^{\prime \prime}\right)$. Если ребро $е$ является петлей с вершиной $W_{i}$, то кольцо обозначим $K_{i i}$, а $\partial K_{i i}$ обозначим через $T_{i i}^{(1)}$ и $T_{i i}^{(2)}$ (если петель несколько, то будем ставить штрихи).

Пусть на $Q^{3}$ задано слоение Лиувилля, которое задается молекулой $G$ (мы предполагаем, что $\left.G=W^{*}\left(Q^{3}\right)\right)$. Разобьем $Q^{3}$ на два подмножества $X_{1}$ и $X_{2}$, где

$$
\begin{aligned}
& X_{1} \stackrel{\text { def }}{=} \coprod_{\{W \neq A\}} W \\
& X_{2} \stackrel{\text { def }}{=}\left(\coprod_{A \in G} A\right) \sqcup\left(\coprod_{\{K \mid K-\text { добавляемое кольцо }\}} K\right) .
\end{aligned}
$$

Триада $\left(Q^{3} ; X_{1}, X_{2}\right)$, очевидно, вырезаемая. Кроме того, имеем:

$$
X_{1} \cap X_{2}=\left(\coprod_{\{e \mid e-\text { внешнее ребро } G\}} T^{2}\right) \sqcup\left(\coprod_{\{e \mid e-\text { внутреннее ребро } G\}}\left(T^{2} \sqcup T^{2}\right)\right) .
$$

Для триады $\left(Q^{3} ; X_{1}, X_{2}\right)$ напишем точную последовательность Майера-Виеториса:

$$
\begin{aligned}
& \stackrel{\partial_{*}}{\longrightarrow} H_{1}\left(X_{1} \cap X_{2}\right) \stackrel{i_{*}}{\longrightarrow} H_{1}\left(X_{1}\right) \oplus H_{1}\left(X_{2}\right) \stackrel{j_{*}}{\longrightarrow} H_{1}\left(Q^{3}\right) \longrightarrow \\
& \longrightarrow H_{0}\left(X_{1} \cap X_{2}\right) \stackrel{i_{*}}{\longrightarrow} H_{0}\left(X_{1}\right) \oplus H_{0}\left(X_{2}\right) \stackrel{j_{*}}{\longrightarrow} H_{0}\left(Q^{3}\right) \longrightarrow 0,
\end{aligned}
$$


где $i_{*}(c)=i_{1 *}(c)-i_{2 *}(c), i_{k}: X_{1} \cap X_{2} \hookrightarrow X_{k}(k=1,2)$, a $j_{*}\left(c_{1}, c_{2}\right)=j_{1 *}\left(c_{1}\right)+$ $j_{2 *}\left(c_{2}\right), j_{k}: X_{k} \hookrightarrow Q^{3}(k=1,2)$.

Очевидно, имеем:

$$
\begin{aligned}
H_{0}\left(X_{1}\right) \cong Z^{n}, \\
H_{0}\left(X_{2}\right) \cong Z^{m}, \\
H_{0}\left(X_{1} \cap X_{2}\right) \cong Z^{p},
\end{aligned}
$$

где

$n=\{$ число вершин $G$ степени $>1\}$,

$m=\{$ число атомов $A\}+\{$ число внутренних ребер $G\}=\{$ число ребер графа $G\}$, $p=\{$ число ребер $G\}+\{$ число внутренних ребер $\}$.

В силу точности имеем цепочку изоморфизмов абелевых групп:

$$
Z \cong H_{0}\left(Q^{3}\right) \cong\left(H_{0}\left(X_{1}\right) \oplus H_{0}\left(X_{2}\right)\right) / \operatorname{Im}\left(i_{*}\right)
$$

$\mathrm{C}$ другой стороны, $\operatorname{Im}\left(\partial_{*}\right)$ является свободной абелевой группой (как подгруппа свободной группы). Сравнивая ранги групп, получаем равенство

$$
1=(n+m)-\left(p-\operatorname{rk}\left(\operatorname{Im}\left(\partial_{*}\right)\right)\right)
$$

откуда получаем, что

$$
\operatorname{Im} \partial_{*} \cong Z^{b_{1}(G)}
$$

где $b_{1}(G)$ - первое число Бетти. Из того, что $\operatorname{Im} \partial_{*}$ является свободной группой, следует неканонический изоморфизм:

$$
\begin{gathered}
H_{1}\left(Q^{3}\right) \cong\left(\operatorname{Im}\left(j_{*}\right)\right) \oplus Z^{b_{1}(G)}, \\
H_{1}\left(Q^{3}\right) \cong\left[\left(H_{1}\left(X_{1}\right) \oplus H_{1}\left(X_{2}\right)\right) / \operatorname{Im}\left(i_{*}\right)\right] \oplus Z^{b_{1}(G)}, \\
i_{*}: H_{1}\left(X_{1} \cap X_{2}\right) \rightarrow H_{1}\left(X_{1}\right) \oplus H_{2}\left(X_{2}\right) .
\end{gathered}
$$

Чтобы понять, как устроен гомоморфизм $i_{*}$ в выбранных нами координатах, рассмотрим сегмент графа $G$, который показан на рис. 4 . Базисы в группах гомологии $H_{1}\left(W_{i}\right)$ и $H_{1}\left(W_{j}\right)$, как договорились раньше, обозначим соответственно

$$
\left(c_{1}^{(i)}, \ldots, c_{q_{i}-1}^{(i)} ; \xi^{(i)} ; h_{1}^{(i)^{\prime}}, \ldots, h_{g_{i}}^{(i)^{\prime \prime}}\right)
$$

и

$$
\left(c_{1}^{(j)}, \ldots, c_{q_{j}-1}^{(j)} ; \xi^{(j)} ; h_{1}^{(j)^{\prime}}, \ldots, h_{g_{j}}^{(j)^{\prime \prime}}\right) .
$$


Тогда $i_{*}$ в координатах имеет вид

$$
\left(\begin{array}{cccccccccccccc}
\beta_{i k} & 0 & \ldots & \alpha_{i k} & 0 & 0 & 0 & \ldots & 0 & 0 & 0 & 0 & 0 & \ldots \\
\delta_{i k} & 0 & \ldots & \gamma_{i k} & 0 & 0 & 0 & \ldots & 0 & 0 & -1 & 0 & 0 & \ldots \\
0 & 0 & \ldots & 1 & \vdots & 0 & 0 & \ldots & 0 & \vdots & 0 & -1 & 0 & \ldots \\
0 & 1 & \ldots & 0 & & 0 & 0 & \ldots & 0 & & 0 & 0 & -(1) & \ldots \\
0 & 0 & \ldots & 0 & 0 & \beta_{i j} & 0 & \ldots & -\delta_{i j} & 0 & 0 & -1 & 0 & \ldots \\
0 & 0 & \ldots & 0 & 0 & -\alpha_{i j} & 0 & \ldots & \gamma_{i j} & 0 & 0 & 0 & -1 & \ldots \\
& & & & & & & & & & & & & \\
& * & & * & * & & * & & * & * & * & & * &
\end{array}\right)
$$

Нашей дальнейшей целью будет диагонализация этой матрицы при помощи целочисленных преобразований над строками и столбцами. Мы ограничимся умножением строки (столбца) на целое $k$ и добавлением к другой строке (столбцу).

Сделаем несколько очевидных замечаний.

1) Циклы $\left(h^{i}\right)$ дают свободные элементы в $H_{1}\left(Q^{3}\right)$. Их число есть

$$
\sum_{W \neq A} 2 g(W)
$$

Таким образом, первая часть теоремы доказана, т.е. доказано неравенство

$$
\operatorname{rk} H_{1}\left(Q^{3}\right) \geqslant b_{1}(G)+2 \sum_{W \neq A} g(W) .
$$

$2)$ Без ограничения общности, число $(-1)$ в столбце $\left(e_{k}\right)$ можно считать матричной единицей. Следовательно, после факторизации цикл $e_{k}$ обращается в 0 (в $\left.H_{1}\left(Q^{3}\right)\right)$.

3) Кроме того, числа (-1) “в кружочках" после вычитания из (-1) расположенных под ними, тоже являются матричными, и, следовательно, матрица упрощается. Отсюда следует грубое неравенство

$$
\operatorname{rk} H_{1}\left(Q^{3}\right) \leqslant\left(b_{1}(G)+2 \sum_{W \neq A} g(W)\right)+\sum_{W \neq A} q(W),
$$

где $q(W)$ - валентность атома $W,\left(\right.$ поскольку $\sum_{W \neq A} q(W)$ есть число столбцов новой матрицы).

После описанных выше упрошений матрица принимает вид

$$
\left(\begin{array}{ccccccccccc}
\beta_{i k} & 0 & \ldots & \alpha_{i k} & 0 & 0 & \ldots & 0 & 0 & \ldots & 0 \\
0 & 0 & \ldots & -1 & \beta_{i j} & 0 & \ldots & -\delta_{i j} & 0 & \ldots & 0 \\
0 & -1 & \ldots & 0 & -\alpha_{i j} & 0 & \ldots & \gamma_{i j} & 0 & \ldots & 0 \\
0 & 0 & & & 0 & 0 & & & & & \\
\vdots & \vdots & & & \vdots & \vdots & & & & &
\end{array}\right)
$$

ОПРЕДЕЛЕНИЕ 5. Эту матрицу мы будем обозначать через $M(G)$ и назьвать характеристической матрицей графа $G$. 


$$
\begin{aligned}
& A_{m_{1}} \backslash \begin{array}{ll}
A_{1} & A_{1} \\
\uparrow \infty & \uparrow r_{21} \nearrow
\end{array} A_{l_{1}} \\
& \vdots \leftarrow W_{1} . \rightarrow . W_{2} \rightarrow \vdots \\
& A_{m_{t}} \quad A_{l_{k}}
\end{aligned}
$$

Рис. 5

ЗАмечАниЕ 3. Нетрудно проверить, что характеристическая матрица является квадратной порядка $\sum_{W \neq A} q(W)$.

Теперь докажем вторую часть теоремы. Для этого рассмотрим ситуацию, показанную на рис. 5 .

Тогда характеристическая матрица $M(G)$ имеет вид

$$
\left(\begin{array}{ccccccccccc}
\beta_{11} & 0 & \ldots & 0 & \alpha_{11} & & & & & \\
0 & \beta_{1 m_{1}} & & \vdots & \alpha_{1 m_{1}} & & 0 & & & \\
\vdots & & \ddots & & \vdots & & & & & & \\
0 & \ldots & & \beta_{1 m_{t}} & \alpha_{1 m_{t}} & & & & & & \ldots \\
0 & 0 & \ldots & 0 & -1 & -\beta_{12} & -\beta_{12} & \ldots & -\beta_{12} & -\delta_{12} & \ldots \\
1 & 1 & \ldots & 1 & 0 & \alpha_{12} & \alpha_{12} & \ldots & \alpha_{12} & \gamma_{12} & \ldots \\
& & & & & \beta_{21} & 0 & & \alpha_{21} & \ldots \\
& 0 & & & & 0 & \beta_{2 l_{1}} & & \alpha_{2 l_{1}} & \cdots \\
& & & & & & & \ddots & \vdots &
\end{array}\right)
$$

На рис. 5 точками “.” обозначен тот кусок края атома, меридиан которого не является базисным элементом выбранного нами базиса группы $H_{1}(W)$. Так как $\beta_{11}=0$, то $\alpha_{11}=\varepsilon_{11}$. Следовательно, можно "уничтожить" все элементы “под" $\alpha_{11}$ в столбце $\left(\xi^{(1)}\right)$. Аналогичным образом, можно “уничтожить" все элементы "справа" от первой единицы в строке $\left(\mu_{12}^{(2)}\right)$. Отсюда уже видно, что $Z_{\beta_{1 m_{1}}} \oplus \cdots \oplus Z_{\beta_{1 m_{t}}}$ будет прямым слагаемым в $H_{1}\left(Q^{3}\right)$.

Если, кроме того, допустим, что $r_{21}=\infty$, то “уничтожая" при помоши $\alpha_{21}=\varepsilon_{21}$ столбец $\left(\xi^{(2)}\right)$ и все справа от первого - $\beta_{12}$, мы видим, что появляется дополнительное слагаемое $Z_{\beta_{12}}$ в группе $H_{1}\left(Q^{3}\right)$. Аналогично рассматриваются остальные случаи сушественных ребер.

Теперь перейдем к доказательству последней части теоремы.

Рассмотрим произвольную молекулу $G$. Согласно ранее доказанному

$$
H_{1}\left(Q^{3}\right) \cong A \oplus Z^{b_{1}(G)} \oplus Z^{\sum_{W \neq A} 2 g(W)},
$$

где

$$
A \cong\left\langle e_{1}, \ldots, e_{k}\right\rangle /\langle M(G) e\rangle
$$


$e_{i}$ - базисные элементы свободной конечнопорожденной абелевой групш, $e-$ столбец $\left[e_{1}, \ldots, e_{k}\right]$, а $M(G)$ - характеристическая матрица молекулы $G$. Определим “полную энергию кручения" молекулы $G$ по следующей формуле:

$$
N(G) \stackrel{\text { def }}{=} \operatorname{det} M(G) \text {. }
$$

Применяя целочисленные и обратимые преобразования над строками и столбцами $M(G)$, мы можем привести ее к диагональному виду. Следовательно, если $\operatorname{rk} A \geqslant 1$, то на диагонали стоит как минимум один нуль, и, значит, $N(G)=0$. Если rk $A=0$, то очевидно, что

$$
N(G)=\operatorname{card}\left(\operatorname{Tor} H_{1}\left(Q^{3}\right)\right)
$$

Заметим, что $N(G)$ для любого фиксированного графа должна быть функцией меток, так как $Q^{3}$ восстанавливается по графу с метками с точностью до гомеоморфизма (см. [2]).

Рассмотрим теперь тот случай, когда молекула содержит звездочки (см. [2]). Пусть $W^{*}$ есть атом, имеющий звездочку, т.е. особый слой типа $(1,2)$ расслоения Зейферта атома $W^{*}$. Предполагаем, что ориентация на 3 -многообразии $Q^{3}$ фиксирована, и тем самьм на атоме $W^{*}$ задана ориентация. Рассмотрим регулярную окрестность особого слоя (топологически это есть просто полноторие $D^{2} \times S^{1}$ ). Обозначим ее через $K$, а замькание ее дополнения в $W$ через $\widetilde{K}$. На граничном торе $T^{2}=\partial K=\partial \widetilde{K}$ рассмотрим два базиса в группе гомологий $H_{1}\left(T^{2}\right):\left(\lambda^{\prime}, \mu^{\prime}\right)$ и $(\lambda, \mu)$. В качестве $\lambda$ выбираем базисньй цикл, однозначно определяемый условием $i_{*}(\lambda)=0$, где $i: T^{2} \hookrightarrow K$, ориентация которого индуцируется естественной ориентацией базы $D^{2}$ полнотория $K$. Если через $\mu_{0}$ обозначим базисный цикл в $H_{1}(K)$, ориентация которого совпадает с ориентацией критической окружности, индуцируемой полем sgrad $H$, то тогда цикл $\mu$ однозначно определяется условиями:

1) $i_{*}(\mu)=2 \mu_{0}$,

2) $\chi=\lambda+2 \mu$,

где $\chi$ есть слой расслоения Зейферта. В качестве $\lambda^{\prime}$ выбираем слой расслоения Зейферта. Тогда цикл $\mu^{\prime}$ однозначно определяется условием, что матрица склейки есть

$$
\left\{\begin{array}{l}
\lambda=\lambda^{\prime}+2 \mu^{\prime}, \\
\mu=-\mu^{\prime} .
\end{array}\right.
$$

Такой цикл $\mu^{\prime}$ будем назьвать допустимым. Обозначим через $\widetilde{W}$ дополнение в атоме $W$ ко всем регулярным окрестностям особых слоев расслоения Зейферта атома $W$.

ОПрЕДЕЛЕниЕ 6 . Сечение расслоения Зейферта в $\widetilde{W}$ называется допустимылм, если на границах регулярных окрестностей особых слоев оно “высекает” допустимые циклы.

Таким образом, случай наличия звездочек очевидным образом сводится к уже доказанному при помощи редукции, которую мы описали перед формулировкой основной теоремы.

Тем самьм теорема полностью доказана. 


\section{4. Применение полученных результатов}

Рассмотрим сначала случай Сретенского движения гиростата в гравитационном поле. Как и в любом другом интегрируемом случае динамики твердого тела, уравнения движения удобно рассмотреть на алгебре Ли $e(3)^{*}$ (группы $E(3)$ движения евклидова 3 -пространства) с пуассоновой структурой, задаваемой формулой

$$
\left\{f_{1}, f_{2}\right\}(x) \stackrel{\text { def }}{=} x\left(\left[\nabla_{x} f_{1}, \nabla_{x} f_{2}\right]\right),
$$

где $\nabla_{x} f_{i} \in e(3)$.

На орбитах коприсоединенного представления эта скобка невырождена и задает симплектическую структуру. В координатах $\left\{\left(R_{i}, S_{i}\right)\right\}$ такая орбита задается уравнением

$$
T S^{2}=\left\{f_{1}=R_{1}^{2}+R_{2}^{2}+R_{3}^{2}=1, f_{2}=R_{1} S_{1}+R_{2} S_{2}+R_{3} S_{3}=g\right\} .
$$

В случае Сретенского $(g=0)$ мы имеем следующие интегралы:

$$
\begin{aligned}
& K=\left(S_{3}+2 \lambda\right)\left(S_{1}^{2}+S_{2}^{2}\right)-S_{1} R_{3}, \\
& H=\frac{1}{2}\left(S_{1}^{2}+S_{2}^{2}+4\left(S_{3}+\lambda\right)^{2}\right)+R_{1} .
\end{aligned}
$$

При $\lambda=0$ получаем случай Горячева-Чаплыгина движения твердого тела с закрепленной точкой.

Бифуркационная диаграмма отображения момента

$$
H \times K: T S^{2} \rightarrow R^{2}
$$

была исследована в [5], а в работе [3] была построена грубая классификация (т.е. был построен граф молекулы).

В зависимости от параметра $\lambda$ имеем четыре случая:
(a) $\lambda=0$,
(b) $-1 / 3<\lambda<0$,
(c) $-1<\lambda<-1 / 3$,
(d) $\lambda<-1$.

В случае (а) мы имеем молекулы (3), (4) (см. таблицу 1). Найдем $n$-метку для первой из них. Из " $n$-соотношения" имеем:

$$
N(G)=2\left(n+1 \cdot \frac{1}{2}\right)= \pm 1 \quad \Rightarrow \quad n=0 \text { или } n=-1 .
$$

Выбирая подходящую ориентацию 3 -многообразия $Q^{3}$, можно считать без ограничения обшности, что $n=0$.

Для второй молекулы этого случая имеем

$$
\begin{gathered}
N(G)=8\left\{\left(n_{1}+\frac{1}{2}+\varepsilon_{1} \cdot \frac{1}{2}\right)\left(n_{2}+\left(\frac{1}{2}-1\right)+\varepsilon_{2} \cdot \frac{1}{2}\right)-\frac{1}{4}\right\}= \pm 2, \\
\left(\left(2 n_{1}+1\right)+\varepsilon_{1}\right)\left(\left(2 n_{2}-1\right)+\varepsilon_{2}\right)=1 \pm 1
\end{gathered}
$$


Слева стоит число вида $4 k$, следовательно,

$$
\left(\left(2 n_{1}+1\right)+\varepsilon_{1}\right)\left(\left(2 n_{2}-1\right)+\varepsilon_{2}\right)=0 .
$$

Здесь мы пользуемся тем фактом, что при замене ориентации 3 -многообразия $Q^{3}$ все $\varepsilon$-метки, которые стоят на внутренних ребрах с $r \neq \infty$, меняют знак. Если ребро внешнее и $r \neq \infty$, то метка $\varepsilon$ знак не меняет. При $r=\infty$ метка $\varepsilon$ меняет знак на внешних ребрах и не меняет знак на внутренних. Таким образом, мы без ограничения общности считаем, что $\varepsilon=1$ на ребрах типа $B \stackrel{r \neq \infty}{\longrightarrow} B$. В дальнейшем мы будем делать это без оговорки.

Рассмотрим отображение $\Phi: e(3)^{*} \rightarrow e(3)^{*}$, задаваемое формулой

$$
\left\{\begin{array}{l}
R_{i} \mapsto R_{i}, \quad S_{i} \mapsto S_{i} \quad \text { при } i=1,2, \\
R_{3} \mapsto-R_{3}, \quad S_{3} \mapsto-S_{3} .
\end{array}\right.
$$

Очевидно, что $\Phi^{*} H=H, \Phi^{*} K=-K, \Phi^{*} \omega=-\omega$. Следовательно,

$$
\Phi_{*}(\operatorname{sgrad} H)=-\operatorname{sgrad} H
$$

С другой стороны, так как $\Phi^{*}(\omega \wedge \omega)=\omega \wedge \omega$, то $\Phi$ сохраняет ориентацию на орбите коприсоединенного действия. С другой стороны, из равенства $\Phi^{*} H=H$ следует, что $\Phi$ сохраняет ориентацию изоэнергетической поверхности. Таким образом, на поверхности $H=$ const отображение $\Phi$ индуцирует послойное отображение, которое сохраняет ориентацию поверхности и меняет ориентацию на всех критических окружностях. Рассмотрим два экземпляра молекулы (4), снабженные всеми метками. На одном из них поменяем ориентацию внутреннего ребра. Нетрудно проверить, что при этом $n$-метки меняются так:

$$
\begin{aligned}
& n_{1} \mapsto n_{1}+1, \\
& n_{2} \mapsto n_{2}-1 .
\end{aligned}
$$

Молекулы, полученные таким образом, должны совпадать. Следовательно, $n_{1}=$ $n_{2}-1$ и $\varepsilon_{1}=\varepsilon_{2}$. Таким образом, получаем:

$$
\left(2 n_{1}+1+\varepsilon\right)^{2}=0 .
$$

Имеем две возможности:

а) $n_{1}=0, n_{2}=1, \varepsilon=-1$,

б) $n_{1}=-1, n_{2}=0, \varepsilon=1$.

В случае (с) имеем молекулы $(1),(3),(4),(5),(7),(8)$ (см. таблицу 1). Рассмотрим сначала молекулу (7). Полные энергии семей таковы:

$$
\begin{aligned}
& E\left(F_{1}\right)=n_{1}+\frac{1}{2}+1 \cdot\left(\frac{1}{2}-1\right)=n_{1}, \\
& E\left(F_{2}\right)=n_{2}+\frac{1}{2}, \\
& E\left(F_{3}\right)=n_{3} .
\end{aligned}
$$


Имеем следующее " $n$-соотношение" :

$$
\begin{gathered}
N(G)=4\left\{n_{1} n_{3}\left(n_{2}+\frac{1}{2}\right)-\frac{1}{4} n_{3}-1 \cdot n_{1}\right\}=0 \\
n_{3}\left\{2 n_{1}\left(2 n_{2}+1\right)-1\right\}=4 n_{1} .
\end{gathered}
$$

Рассмотрим два случая.

I) $n_{1}=0 \Rightarrow n_{3}=0$,

откуда получаем решение $n_{1}=0, n_{2}=k, n_{3}=0$.

II) $n_{1} \neq 0 \Rightarrow n_{3} \neq 0$.

Здесь, очевидно, имеем следующие две возможности:

а) $\left\{\begin{array}{l}n_{3}=4 n_{1}, \\ 2 n_{1}\left(2 n_{2}+1\right)-1=1 .\end{array}\right.$

Таким образом, получаем две пары решений:

$n_{1}=1, n_{2}=0, n_{3}=4$ и $n_{1}=-1, n_{2}=-1, n_{3}=-4$.

б) $\left\{\begin{array}{l}n_{3}=-4 n_{1}, \\ 2 n_{1}\left(2 n_{2}+1\right)-1=-1\end{array}\right.$ но эта система несовместна.

Рассмотрим теперь молекулу (8). Из “n-соотношения" получаем:

$$
N(G)=4\left\{n_{1} n_{3}\left(n_{2}+\frac{1}{2}\right)-\frac{1}{4} n_{3}-n_{1}\right\}= \pm 2
$$

Находим пять возможных случаев:
a) $n_{1}=0, n_{2}=k, n_{3}= \pm 2$,
б) $n_{1}=k, n_{2}=-1, n_{3}=-2$,
в) $n_{1}=k, n_{2}=0, n_{3}=2$,
г) $n_{1}=1, n_{2}=0, n_{3}=-6$,
д) $n_{1}=-1, n_{2}=-1, n_{3}=-6$.

Из вида бифуркационной диаграммы следует, что $n_{1}$ и $n_{2}-$ метки молекул (7) и (8) должны совпадать. В результате получаем то, что показано в таблице 1.

Остальные случаи, такие как случай Жуковского движения гиростата в гравитационном поле и случай Ковалевской динамики твердого тела, рассматриваются аналогично. Все результаты показаны в таблицах, приведенных в конце настоящей работы.

Автор выражает благодарность А. Т. Фоменко и А. В. Болсинову за постоянное внимание к работе. 


\begin{tabular}{|c|c|c|c|}
\hline № & молекула & метки & $Q^{3}$ \\
\hline (1) & $A-A$ & & $S^{3}$ \\
\hline (2) & $A-B<_{\infty}^{\infty} A$ & & $S^{1} \times S^{2}$ \\
\hline (3) & 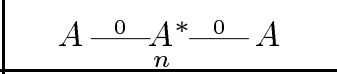 & $n=-1$ & $S^{3}$ \\
\hline (4) & $A \stackrel{1 / 2}{0^{\varepsilon}} \underset{n_{1}}{ } \frac{1 / 2}{\varepsilon_{1}=1} \underset{n_{2}}{B} \stackrel{1 / 2}{\varepsilon}, A_{A}^{0} A$ & $\begin{array}{l}\text { а) } \varepsilon=1, n_{1}=-1, n_{2}=0 \\
\text { б) } \varepsilon=-1, n_{1}=0, n_{2}=1\end{array}$ & $\mathbb{R P}^{3}$ \\
\hline (5) & 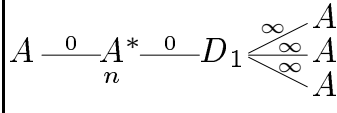 & $n=0$ & $S^{1} \times S^{2} \# S^{1} \times S^{2}$ \\
\hline (6) & $A \underset{n}{A^{*} \stackrel{0}{-} D_{1} \sum_{0}^{\infty} \underset{A}{A} A}$ & $n=0$ & $S^{3}$ \\
\hline (7) & 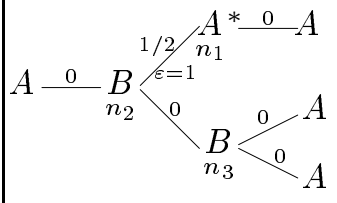 & $\begin{array}{l}\text { а) } n_{1}=0, n_{2}=k, n_{3}=0 \\
\text { б) } n_{1}=1, n_{2}=0, n_{3}=4 \\
\text { в) } n_{1}=-1, n_{2}=-1, n_{3}=-4\end{array}$ & $S^{1} \times S^{2}$ \\
\hline (8) & 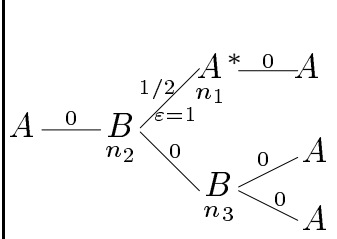 & $\begin{array}{l}\text { а) } n_{1}=0, n_{2}=k, n_{3}= \pm 2 \\
\text { б) } n_{1}=k, n_{2}=-1, n_{3}=-2 \\
\text { в) } n_{1}=k, n_{2}=0, n_{3}=2 \\
\text { г) } n_{1}=1, n_{2}=0, n_{3}=-6 \\
\text { д) } n_{1}=-1, n_{2}=-1, n_{3}=-6\end{array}$ & $\mathbb{R P}^{3}$ \\
\hline
\end{tabular}

ТАБлицА 1. Случай Сретенского 


\begin{tabular}{|c|c|c|c|}
\hline № & молекула & метки & $Q^{3}$ \\
\hline (1) & $A \stackrel{0}{A} A$ & & $S^{3}$ \\
\hline (2) & $A \stackrel{\infty}{\longleftarrow} A$ & & $S^{1} \times S^{2}$ \\
\hline (3) & 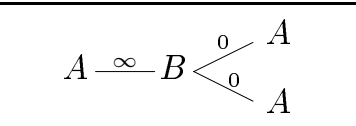 & & $S^{3}$ \\
\hline (4) & 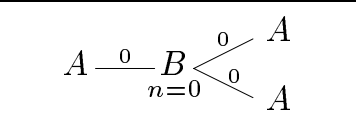 & $n=0$ & $S^{1} \times S^{2}$ \\
\hline (5) & ${ }_{A}^{A} \searrow^{0} B \stackrel{\infty}{\rightleftharpoons} B \stackrel{0}{0^{0}} A$ & & $S^{3}$ \\
\hline (6) & $A^{0} B \frac{\infty}{n=2} B \stackrel{0}{0} A_{A}^{0} A$ & $n=2$ & $\mathbb{R} \mathbb{P}^{3}$ \\
\hline (7) & $A \underbrace{0} B \frac{\infty}{n=0} B \stackrel{0}{0} A_{A}^{A}$ & $n=0$ & $S^{1} \times S^{2}$ \\
\hline
\end{tabular}

ТАБЛИцА 2. Случай Жуковского 


\begin{tabular}{|c|c|c|c|}
\hline № & молекула & метки & $Q^{3}$ \\
\hline$a_{1}$ & $A-A$ & & $S^{3}$ \\
\hline$a_{2}$ & $\begin{array}{l}A \backslash 0 \\
A \stackrel{0}{A_{n_{1}}-0}{ }_{n_{2}} \sum^{0}{ }_{A}\end{array}$ & $\begin{array}{l}n_{1}=1, n_{2}=2 \\
n_{1}=0, n_{2}=k\end{array}$ & $S^{3}$ \\
\hline$a_{3}$ & 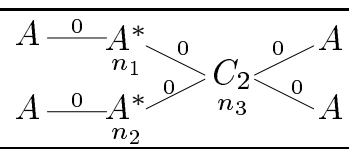 & $n_{1}=0, n_{2}=0, n_{3}=2,6$ & $\mathbb{R P}^{3}$ \\
\hline$b_{2}$ & $A^{0}{ }_{n}^{0} B \frac{1 / 2}{} A$ & $n=0$ & $S^{3}$ \\
\hline$d_{4}$ & 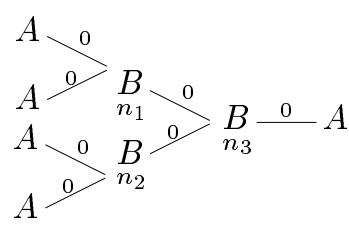 & $\begin{array}{l}n_{1}=1, n_{2}=1, n_{3}=2 \\
n_{1}=0, n_{2}=0, n_{3}=2,6\end{array}$ & $S^{1} \times S^{2}$ \\
\hline$d_{6}$ & 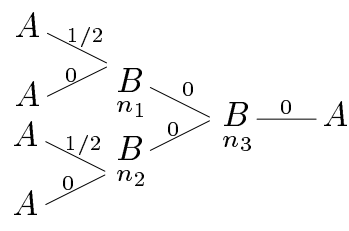 & $n_{1}=0, n_{2}=0, n_{3}=2,6$ & $\mathbb{R} \mathbb{P}^{3}$ \\
\hline$d_{3}$ & $\begin{array}{l}A_{{ }^{\infty}} \\
A^{\infty} B \bigcup_{n}^{0} \\
A_{\bigcup_{\infty}} B \\
A^{\infty}\end{array}$ & $n=0$ & $S^{1} \times S^{2} \# S^{1} \times S^{2}$ \\
\hline
\end{tabular}

ТАБлицА 3. Случай Ковалевской 


\section{Список литературы}

1. Фоменко A. T., Цишанг $X$. Критерий топологической эквивалентности интегрируемых гамильтоновых систем с двумя степенями свободы // Изв. АН СССР. 1990. Т. 54. № 3. C. $546-575$.

2. Болсинов A.B., Матвеев C.В., Фоменко A.T. Топологическая классификация интегрируемых гамильтоновых систем с двумя степенями свободы // УМН. 1990. Т. 45. № 2. C. 49-78.

3. Oshemkov A. A. Fomenko Invariants for the Main Integrable cases of the Rigid Body Motion Equations // AMS. 1991. V. 4. P. 67-146.

4. Bolsinov A. V. Methods of Calculation of the Fomenko-Zieschang Invariants // AMS. 1991. V. 6. P. 147-183.

5. Харламов М. П. Топологический анализ интегрируемых задач в динамике твердого тела. Л.: Изд-во Ленингр. ун-та, 1988.

Московский государственньй

университет им. М.В. Ломоносова
Поступила в редакцию 20.06.1995 